\title{
Optimization of the Size of Dstatcom System Based on Data - Driven Control Design
}

\author{
K.Sukraj, T.Yuvaraj, R. Hariharan, M. Thirumalai
}

\begin{abstract}
This paper elaborates on a Distributed Static Synchronous Compensator (DSTATCOM). It renders a setup of hardware which provides compensation of power along with the optimization of the size of DSTATCOM set up. This DSTATCOM hardware set up reduces the power loss through compensation of power, regulates the voltage profile and increases the power factor. The performance of the DSTATCOM for linear and nonlinear loads was simulated.
\end{abstract}

Keywords: DSTATCOM, Optimization Data Driven Control, Linear and Non-linear Loads.

\section{INTRODUCTION}

Transformer is the input source which provides power supply to the entire grid whereas Regulated power supply (RPS) provides supply to gate driver, Controller, Zero cross detector (ZCD) and Signal conditioner. Rectifier board is available in this hardware setup which has capacitor and a switch and through it's ON and OFF operation and load is converted to linear or non-linear load. The load is high voltage resistor load and it is of the shape of rod and brown colour in the hardware set up and it is of the rating of 50ohm which has the capacity to withstand 50 watt power. In this hardware set up inductive filter is used which is used to convert step wave to sine wave. Five-level invertor is used in this set up and it has MOSFET. Through signal conditional sensing controller circuit gets input voltage and Zero cross detector provides (ZCD) provides frequency of input. Potential transformer provides voltage value to the signal conditioner whereas the current transformer provides current value to the transformer. Since the value of current is less only resistor is used as the current transformer in the hardware set up $[1,2,5]$.

\section{HARDWARE SPECIFICATIONS AND ITS OPERATION}

Figure 1 shows the hardware model of proposed DSTATCOM System Based on Data Driven Control

Revised Manuscript Received on October 14, 2019.

K.Sukraj, P.G. Scholar, Department of Electrical and Electronics Engineering, Saveetha School of Engineering, Saveetha Institute of Medical and Technical Sciences, Chennai, Tamilnadu, India.

(E-mail: sukrajk@gmail.com)

T.Yuvaraj, Assistant Professor, Department of Electrical and Electronics Engineering, Saveetha School of Engineering, Saveetha Institute of Medical and Technical Sciences, Chennai, Tamilnadu, India

(E-mail: yuvaraj4252@gmail.com)

R. Hariharan, Assistant Professor, Department of Electrical and Electronics Engineering, Saveetha School of Engineering, Saveetha Institute of Medical and Technical Sciences, Chennai, Tamilnadu, India.

(E-mail: harinov22@gmail.com)

M. Thirumalai, Assistant Professor, Department of electronics and communication engineering, Saveetha Engineering College, Chennai, Tamilnadu, India.

(E-mail: thirumalai3788@gmail.com)
Design. The explanation of main parts of the model has given below [3,4].

Transformer: It is the input source which supplies power to the entire grid.

Load: High voltage resistor load is used with the specification of $50 \mathrm{ohm}$ and capable of withstanding 50 watt power.

Rectifier Board: It consists of capacitor and a switch by means of $\mathrm{ON}$ and OFF operation of switch the load is converted to linear or non-linear load.

Five - Level Inverter: It has MOSFET which consists of inverter and capacitor.

Controller Circuit: It is a combination of signal conditioner and zero cross detector (ZCD). Signal conditioner senses the signal and it provides input voltage and current whereas zero cross detector (ZCD) provides frequency of input. This controller provides control signal to eight switches on the basis inference from signal conditioner and zero cross detector (ZCD).

Regulated power supply (RPS) : It provides power supply to the entire DSATCOM set up.

Inductive Filter : It converts step wave to sine wave.

Potential Transformer : It provides voltage value to signal conditioner.

Current Transformer : It provides current value to signal conditioner.

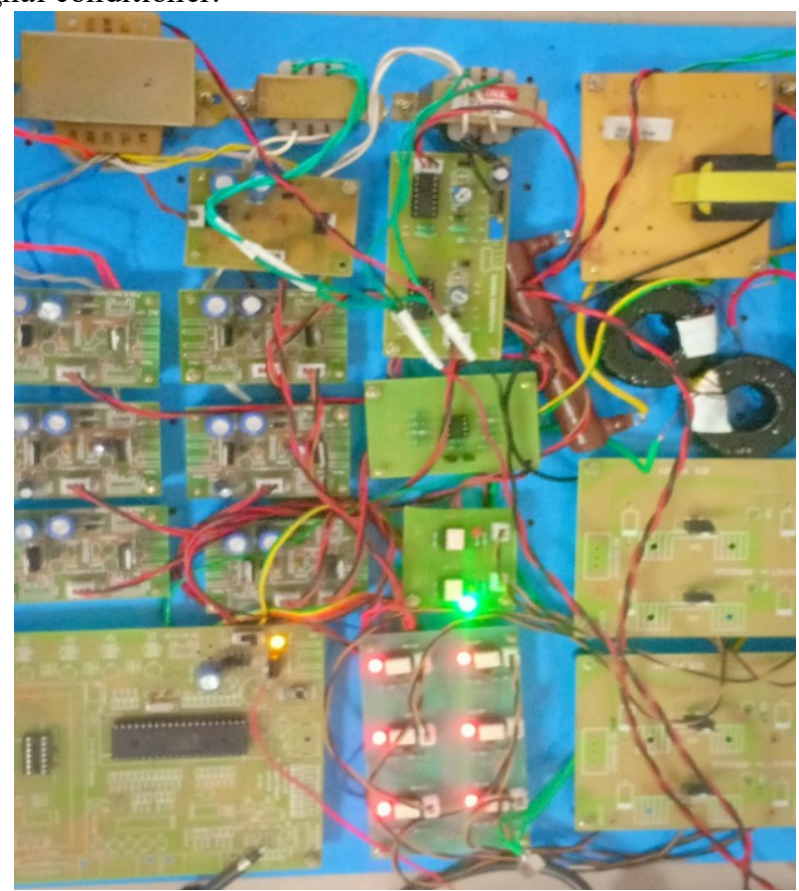

Fig 1: Hardware circuit model

Published By: Blue Eyes Intelligence Engineering \& Sciences Publication 


\section{RESULTS AND DISCUSSION}

\subsection{Linear Load:}

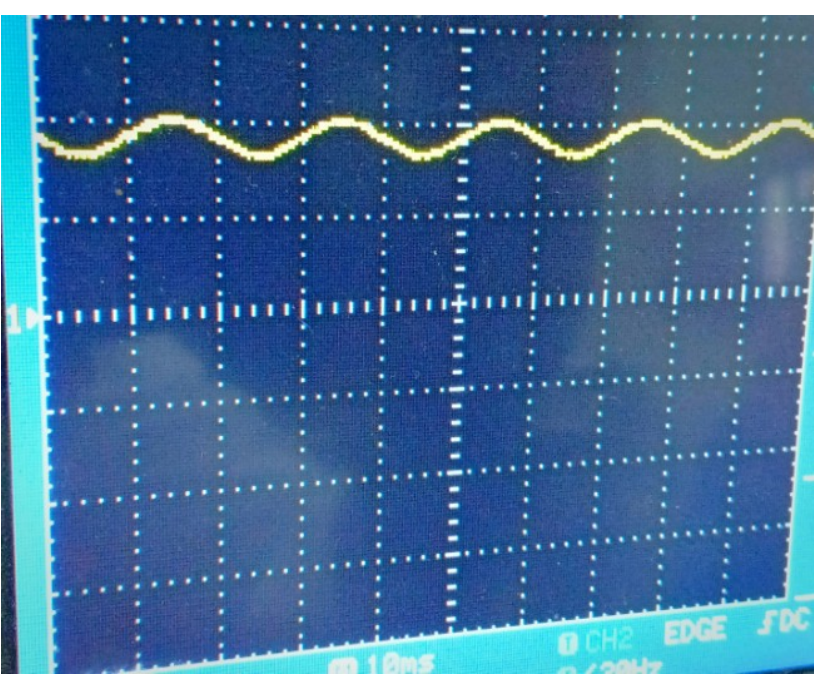

Fig 2: Linear load waveform

The above figure 2 waveform is the linear load which is obtained due to the ON operation of the switch which is available in the capacitor.

\subsection{Non - Linear Load:}

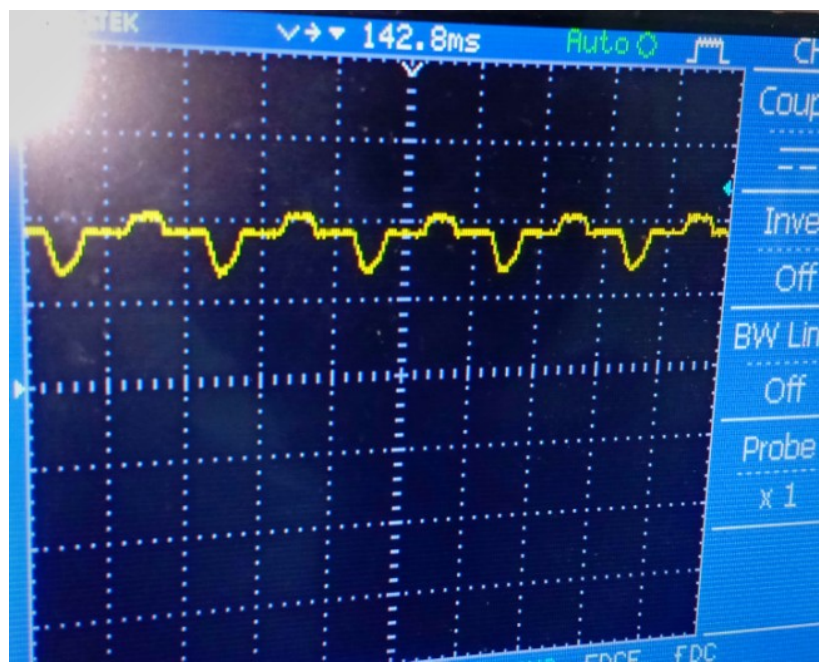

Fig 3: Nonlinear load waveform

The above figure 3 is the non-linear load which is obtained due to the OFF operation of the switch which is available in the capacitor.

\subsection{Output of Zero Cross Detector (ZCD)}

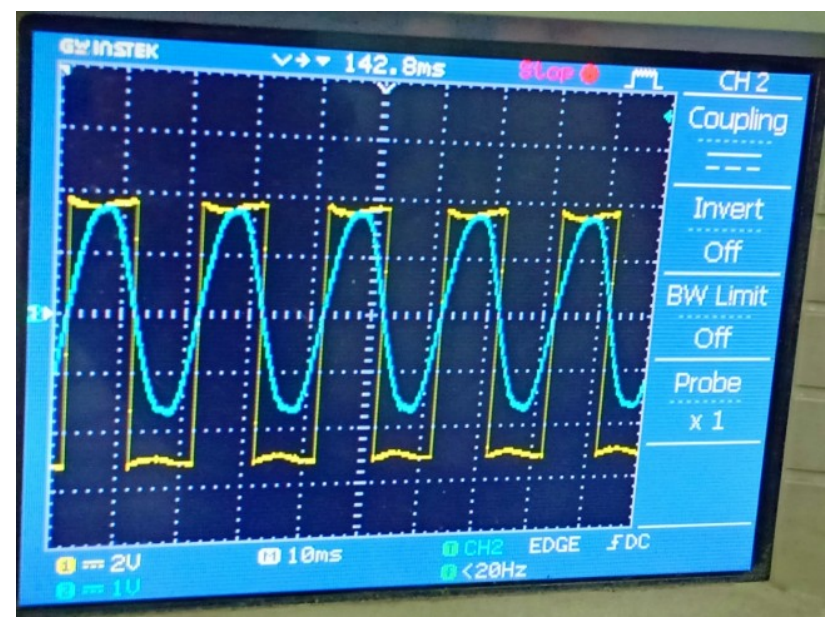

Fig 4: Output of Zero Cross Detector
In the above figure 4 shows the waveform of Zero Cross Detector and blue line represents the input from potential transformer and the yellow line represents the output which is obtained after sensing by zero cross detector(ZCD).

\subsection{Switch Pulse}

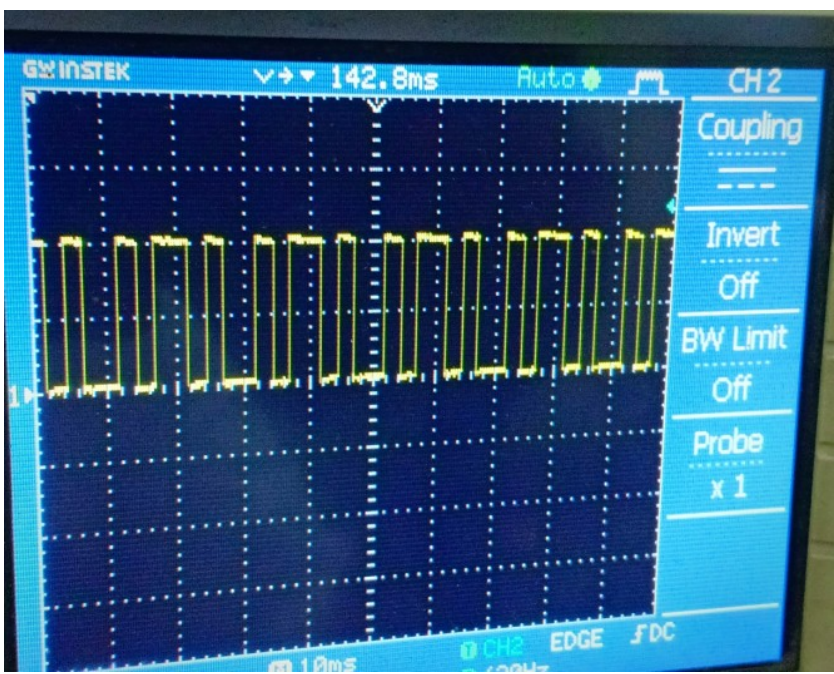

Fig 5: Switch pulse of MOSFET.

The above figure 5 shows the switch pulse of MOSFET.

\section{CONCLUSION}

DSTATCOM provides compensation of power into the power system which ultimately result in the reduction of losses in the network, regulation of voltage profile and the increased the power factor. In this paper various performance of linear and non-linear loads was simulated. The obtained simulated results are found convincing and proves that the compensation is very effective and it also enhances voltage regulation and considerable improvement in power of the power system. Moreover, the size of the DSTATCOM was also optimized.

\section{REFERENCE}

1. Yuvaraj, T., K. Ravi, and K. R. Devabalaji "DSTATCOM allocation in distribution networks considering load variations using bat algorithm", Ain Shams Engineering Journal 8.3 (2017): 391-403.

2. Yuvaraj, T., K. R. Devabalaji, and K. Ravi "Optimal placement and sizing of DSTATCOM using harmony search algorithm", Energy Procedia 79 (2015): 759-765.

3. N. N. V. Surendra Babu, and B.G. Fernandes, "Cascaded Two Level Inverter- Based Multilevel ST ATCOM for High-Power Applications," IEEEE Trans. Power Delivery., vol. 29, no. 3, pp. 993-1001, June. 2014.

4. Hirofumi Akagi, Shigenori Inoue and Tsurugi Yoshii, "Control and Performance of a Transformerless Cascade PWM STATCOM With Star Contiguration," IEEE Trans. Ind. Appl., vol. 43, no. 4, pp. 1041-1049, July/ August 2007.

5. Devabalaji, K. R., et al. "Power loss minimization in radial distribution system", Energy Procedia 79 (2015): 917-923.

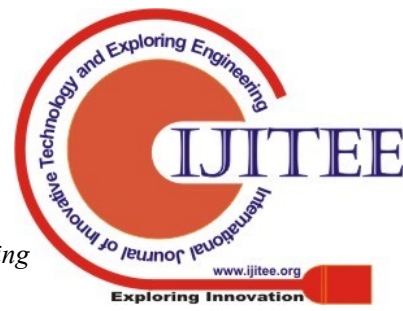

